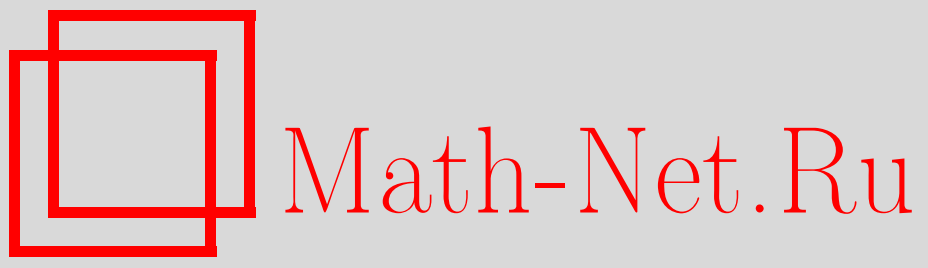

Ю. С. Мишура, Я. А. Ольцик, Выбор оптимального момента переключения на финансовом рынке с альтернативными стратегиями (полумартингальный подход), Теория вероятн. и ее примен., 2000, том 45, выпуск 3, 505-520

DOI: https://doi.org/10.4213/tvp482

Использование Общероссийского математического портала Math-Net.Ru подразумевает, что вы прочитали и согласны с пользовательским соглашением http://www.mathnet.ru/rus/agreement

Параметры загрузки:

IP : 54.224 .187 .69

26 апреля 2023 г., 12:07:58

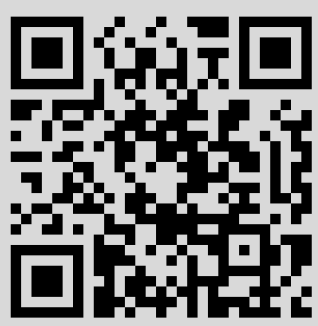




\title{
ВЫБОР ОПТИМАЛЬНОГО МОМЕНТА ПЕРЕКЛЮЧЕНИЯ НА ФИНАНСОВОМ РЫНКЕ С АЛЬТЕРНАТИВНЫМИ СТРАТЕГИЯМИ (ПОЛУМАРТИНГАЛЬНЫЙ ПОДХОД)
}

\begin{abstract}
Пусть инвестор оперирует на финансовом рынке с облигацией и акцией и имеет возможность на фиксированном промежутке времени $[0, T]$ один раз сделать выбор, т.е. переключение, между двумя альтернативными портфелями. Предполагается, что смена финансового портфеля описывается линейным стохастическим дифференциальным уравнением. Оптимальный момент переключения ищется с помощью теории «переломных》 моментов остановки.

Ключевые слова и фразы: случайный процесс, оптимальный момент остановки, линейное стохастическое дифференциальное уравнение, инвестор, капитал.
\end{abstract}

1. Введение. Рассмотрим инвестора, оперирующего на финансовом рынке, который имеет возможность за счет своих средств создать портфель, состоящий из акций и облигаций. Пусть инвестор имеет некоторый начальный капитал $X_{0}$, оперирует на фиксированном промежутке времени $[0, T]$ и имеет возможность не более одного раза перераспределить свой капитал: реализовать имеющиеся активы и на полученные средства создать новый портфель, уплачивая при этом некоторую цену за переключение. Задача инвестора состоит в том, чтобы найти оптимальный момент $\tau \in[0, T]$, в который следует переключиться, так чтобы максимизировать свой средний капитал в конечный момент. Пусть $X_{t}$ - капитал инвестора в момент $t \in[\tau, T]$. Тогда целью является максимизация величины $\mathbf{E} X_{T}$.

Для отыскания оптимального момента переключения вводится понятие «переломного» момента остановки. Он определяется как такой момент, в который субмартингальное свойство некоторого процесса, связанного с капиталом, меняется на супермартингальное. В п. 2 рассматриваются некоторые свойства переломных моментов остановки, а также доказывается, что переломный момент, если такой существует, является оптимальным. В п. 3 ишется оптимальный момент переключения для

\footnotetext{
"Киевский университет им. Т. Шевченко, механико-математический факультет, ул. Владимирская, 64, 252601 Киев, Украина; e-mail: myus@mechmat.univ.kiev.ua, yana@mechmat.univ.kiev.ua
} 
инвестора. В случае, когда цена выбора пропорциональна капиталу в момент выбора, задача решается с помошью переломных моментов остановки. В случае, когда цена включает в себя компоненту, не зависяшую от величины капитала, оптимальный момент переключения ищется с применением формулы Ито.

2. Переломные и оптимальные моменты остановки на конечном промехутке времени. Пусть $(\Omega, \mathscr{F}, \mathbf{P})$ - полное вероятностное пространство, $\left\{\mathscr{F}_{t}, t \in[0, T]\right\}$ - возрастающий непрерывный справа поток $\sigma$-алгебр, $\mathscr{F}_{t} \subset \mathscr{F}$. Пусть $Y_{t}$ - некоторый $\left\{\mathscr{F}_{t}\right\}$-согласованный непрерывный неотрицательный процесс, $t \in[0, T]$. Введем определение и рассмотрим некоторые свойства переломных моментов остановки.

О п р е д е л е н и е 1. Момент остановки (м.о.) $\sigma_{0} \in[0, T]$ называется оптимальным для процесса $Y_{t}$, если

$$
\mathbf{E} Y_{\sigma_{0}}=\sup _{\substack{\tau \in[0, T] \\ \tau-\mathbf{m}, 0 .}} \mathbf{E} Y_{\tau}
$$

Огибающая Снелла [1] для процесса $Y_{t}$ определяется как

$$
f_{t}=\underset{\substack{t \leq \tau \leq T \\ \tau-\mathbf{u . o} .}}{\operatorname{ess} \sup } \mathbf{E}\left(Y_{\tau} \mid \mathscr{F}_{t}\right) .
$$

Из [2] известно, что при выполнении условия $\mathbf{E} \sup _{t \in[0, T]} Y_{t}<\infty$ момент остановки $\sigma=\inf \left\{t \in[0, T]: Y_{t}=f_{t}\right\}$ является оптимальным. Назовем этот момент $\sigma$ минимальным. Заметим, что на конечном отрезке времени минимальный момент остановки всегда существует, поскольку $Y_{T}=f_{T}$.

Все рассматриваемые процессы будем считать согласованными и непрерывными.

Докажем некоторые вспомогательные утверждения, касаюшиеся оптимальных моментов остановки, а также представляющие самостоятельный интерес. Вначале рассмотрим случайные процессы, имеющие супермартингальное свойство «после» некоторого момента остановки $\theta_{1}$, и докажем в леммах 1 и 2 , что $\sigma \leqslant \theta_{1}$ п.н.

Пусть $0 \leqslant t_{1} \leqslant t_{2} \leqslant \cdots \leqslant t_{N} \leqslant T$ - некоторое разбиение отрезка $[0, T]$.

Лемма 1. Пусть $\left\{\xi_{t_{n}}, \mathscr{F}_{t_{n}}, 0 \leqslant n \leqslant N\right\}-$ некоторый $\left\{\mathscr{F}_{t_{n}}\right\}$-согласованный прочесс с дискретнын временем, $\mathbf{E}\left|\xi_{t_{n}}\right|<\infty, 0 \leqslant \theta_{1} \leqslant t_{N}-$ некоторый момент остановки относительно потока $\sigma$-алгебр $\left\{\mathscr{F}_{t_{n}}\right.$, $0 \leqslant n \leqslant N\}$ и выполняется соотношение

$$
\mathbf{E}\left(\xi_{t_{n}} \mid \mathscr{F}_{t_{k}}\right) I\left\{t_{l}=\theta_{1}\right\} \leqslant \xi_{t_{k}} I\left\{t_{l}=\theta_{1}\right\}
$$


$0 \leqslant l \leqslant k \leqslant n \leqslant N$. Тогда для любого $0 \leqslant l \leqslant N$ и для любых моментов остановки $\nu, \tau$ относительно потока $\left\{\mathscr{F}_{t_{n}}\right\}$ таких, ито $0 \leqslant t_{l} \leqslant \nu \leqslant$ $\tau \leqslant t_{N}$, имеет место неравенство

$$
\mathbf{E}\left(\xi_{\tau} \mid \mathscr{F}_{\nu}\right) I\left\{t_{l}=\theta_{1}\right\} \leqslant \xi_{\nu} I\left\{t_{l}=\theta_{1}\right\} \quad \text { n.H. }
$$

Доказательство следует из того факта, что при выполнении соотношения (1) случайный процесс с дискретным временем $\zeta_{t_{n}}=\xi_{t_{n}} I\left\{t_{l}=\right.$ $\left.\theta_{1}\right\}, l \leqslant n \leqslant N$, при фиксированном $l$ является супермартингалом, а также из того, что событие $\left\{\theta_{1}=t_{l}\right\} \in \mathscr{F}_{\theta_{1}} \subset \mathscr{F}_{\nu}$.

Лемма 2. Пусть $\left\{\xi_{t}, \mathscr{F}_{t}, t \in[0, T]\right\}-$ неотрииательный непрерывный случайный прочесс, для которого

$$
\mathbf{E} \sup _{t \in[0, T]} \xi_{t}<\infty,
$$

$\theta_{1} \in[0, T]-$ тахой момент остановки, что для любых $0 \leqslant s_{1} \leqslant s_{2} \leqslant$ $s_{3} \leqslant s_{4} \leqslant T$

$$
\mathbf{E}\left(\xi_{s_{4}} \mid \mathscr{F}_{s_{3}}\right) I\left\{s_{1} \leqslant \theta_{1}<s_{2}\right\} \leqslant \xi_{s_{3}} I\left\{s_{1} \leqslant \theta_{1}<s_{2}\right\} \quad \text { n.н. }
$$

Тогда $\mathbf{P}\left\{\sigma>\theta_{1}\right\}=0$, где $\sigma \in[0, T]-$ минимальный момент остановки dлs прочесса $\xi$.

Д ок а з а т е л с т в о. Введем последовательность дискретных моментов остановки

$$
\sigma_{n}=\left\{\begin{array}{l}
\frac{i}{2^{n}} T, \quad \frac{i-1}{2^{n}} T \leqslant \theta_{1}<\frac{i}{2^{n}} T, 1 \leqslant i \leqslant 2^{n}, n \geqslant 1, \\
T, \quad \theta_{1}=T .
\end{array}\right.
$$

Тогда

$$
\begin{aligned}
& \mathbf{P}\left\{\sigma>\theta_{1}\right\}=\lim _{n \rightarrow \infty} \mathbf{P}\left\{\sigma>\sigma_{n}\right\}=\lim _{n \rightarrow \infty} \sum_{i=1}^{2^{n}-1} \mathbf{P}\left\{\sigma>\frac{i}{2^{n}} T, \sigma_{n}=\frac{i}{2^{n}} T\right\}
\end{aligned}
$$

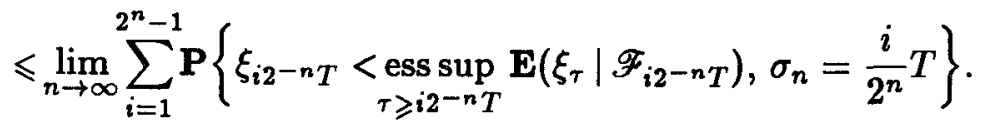

Если $\tau \geqslant i 2^{-n} T$ принимает конечное число значений, то в силу леммы 1 и соотношения (3)

$$
\mathbf{E}\left(\xi_{\tau} \mid \mathscr{F}_{i 2^{-n} T}\right) I\left\{\frac{i}{2^{n}} T=\sigma_{n}\right\} \leqslant \xi_{i 2^{-n} T} I\left\{\frac{i}{2^{n}} T=\sigma_{n}\right\} \text { п.н. }
$$

Если $\tau \in\left[i 2^{-n} T, T\right]-$ произвольный момент остановки, то рассмотрим последовательность моментов остановки $\left\{\tau_{k}, k \geqslant 1\right\}$ :

$$
\tau_{k}= \begin{cases}\frac{i}{2^{n}} T+\frac{j}{2^{k}} T\left(1-\frac{i}{2^{n}}\right), & \frac{i}{2^{n}} T+\frac{j-1}{2^{k}} T\left(1-\frac{i}{2^{n}}\right) \leqslant \tau \\ & <\frac{j}{2^{k}} T\left(1-\frac{i}{2^{n}}\right), \quad j=1, \ldots, 2^{k}, \\ \tau, & \tau .\end{cases}
$$


Tогда $\tau_{1} \geqslant \tau_{2} \geqslant \cdots \geqslant \tau \geqslant \sigma_{n}=i 2^{-n} T$, и в силу леммы 1

$$
\mathbf{E}\left(\xi_{\tau_{k}} \mid \mathscr{F}_{i 2^{-n} T}\right) I\left\{\frac{i}{2^{n}} T=\sigma_{n}\right\} \leqslant \xi_{i 2^{-n} T} I\left\{\frac{i}{2^{n}} T=\sigma_{n}\right\} \text { п.н. }
$$

При $k \rightarrow \infty \xi_{\tau_{k}} \rightarrow \xi_{\tau}$ п.н. Кроме того, в силу условия (2) $\xi_{\tau_{k}}$ является равномерно интегрируемой, и из (5)

$$
\mathbf{E}\left(\xi_{\tau} \mid \mathscr{F}_{i 2-n}\right) I\left\{\frac{i}{2^{n}} T=\sigma_{n}\right\} \leqslant \xi_{i 2^{-n} T} I\left\{\frac{i}{2^{n}} T=\sigma_{n}\right\} \text { п.н. }
$$

Поэтому, согласно определению существенного супремума,

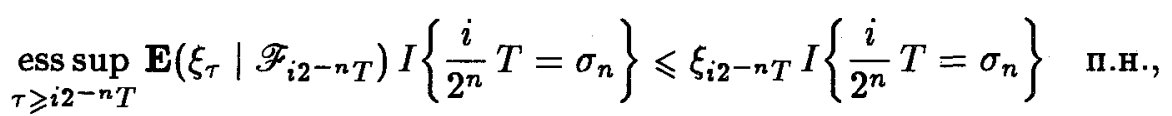

а тогда правая часть (4) равна нулю, и лемма доказана.

Теперь рассмотрим случайные процессы, имеющие субмартингальное свойство «до» некоторого момента остановки $\theta_{2}$, и докажем в леммах $3-5$, что $\sigma \geqslant \theta_{2}$ п.н.

Лемма 3. Пусть $\left\{\xi_{t_{n}}, \mathscr{F}_{t_{n}}, 0 \leqslant n \leqslant N\right\}-$ неотричательный $\left\{\mathscr{F}_{t_{n}}\right\}$-согласованный прочесс $c$ дискретным временем, $\mathbf{E} \xi_{t_{n}}<\infty$. Iусть также моменты остановки $0 \leqslant \sigma_{1} \leqslant \sigma_{2} \leqslant t_{N}$ относительно потока $\left\{\mathscr{F}_{t_{n}}, 0 \leqslant n \leqslant N\right\}$ и семейство $\zeta\left(t_{l}, t_{n}\right), 0 \leqslant l<n \leqslant N, \mathscr{F}_{t_{l}}$-измеримых неотричательных ограниченных ө совокупности случайных величин удовлетворяют соотношению $\zeta\left(t_{l}, t_{n}\right) \mathbf{E}\left(\xi_{t_{n}} \mid \mathscr{F}_{t_{l}}\right) I\left\{\sigma_{1}=t_{k} \leqslant t_{l}<\right.$ $\left.t_{n} \leqslant \sigma_{2}\right\} \geqslant \xi_{t_{l}} I\left\{\sigma_{1}=t_{k} \leqslant t_{l}<t_{n} \leqslant \sigma_{2}\right\}$ n.н., $0 \leqslant k \leqslant l<n \leqslant N$. Tozda

$$
\begin{aligned}
& \mathbf{E}\left(\prod_{k=1}^{N-1} \zeta\left(t_{k}, t_{k+1}\right) I\left\{\sigma_{1} \leqslant t_{k}<t_{k+1} \leqslant \sigma_{2}\right\} \xi_{\sigma_{2}} \mid \mathscr{F}_{\sigma_{1}}\right) I\left\{\sigma_{1}<\sigma_{2}\right\} \\
& \quad \geqslant \xi_{\sigma_{1}} I\left\{\sigma_{1}<\sigma_{2}\right\} \quad \text { n.r. }
\end{aligned}
$$

Д ок аз а т л в с т в о. Очевидно, случайные величины $I\left\{\sigma_{1}<\right.$ $\left.\sigma_{2}\right\}$ и $\mathbf{E}\left(\xi_{\sigma_{2}} \mid \mathscr{F}_{\sigma_{1}}\right)$ являются $\mathscr{F}_{\sigma_{1}}$-измеримыми, $\left\{\sigma_{1}<\sigma_{2}\right\} \cap\left\{\sigma_{1}=t_{k}\right\} \in$ $\mathscr{F}_{t_{k}}$ для любого $0 \leqslant k \leqslant N$.

Пусть $A \in \mathscr{F}_{\sigma_{1}}$ - произвольное событие. Обозначим $\eta_{k}(s, t)=$ $\zeta\left(t_{k}, t_{k+1}\right) I\left\{s=t_{i}<t_{k+1} \leqslant t\right\}, A_{k}=\left\{\sigma_{1}=t_{i}<t_{k+1} \leqslant \sigma_{2}\right\}$, $k=i, \ldots, N-1, i=0, \ldots, N-1$. Тогда

$$
\begin{aligned}
& \int_{A \cap\left\{\sigma_{1}<\sigma_{2}\right\}}\left(\prod_{k=1}^{N-1} \eta_{k}\left(\sigma_{1}, \sigma_{2}\right) \xi_{\sigma_{2}}-\xi_{\sigma_{1}}\right) d \mathbf{P} \\
& =\sum_{i=0}^{N-1} \int_{A \cap\left\{\sigma_{1}=t_{i}<\sigma_{2}\right\}}\left(\prod_{k=i}^{N-1} \eta_{k}\left(t_{i}, \sigma_{2}\right) \xi_{\sigma_{2}}-\xi_{t_{i}}\right) d \mathbf{P}
\end{aligned}
$$




$$
\begin{aligned}
& \geqslant \sum_{i=0}^{N-1} \int_{A \cap A_{i}}\left(\prod_{k=i}^{N-1} \eta_{k}\left(t_{i}, \sigma_{2}\right) \xi_{\sigma_{2}}-\eta_{i}\left(t_{i}, \sigma_{2}\right) \xi_{t_{i+1}}\right) d \mathbf{P} \geqslant \cdots \\
& \geqslant \sum_{i=0}^{N-1} \int_{A \cap A_{N-1}}\left(\prod_{k=i}^{N-1} \eta_{k}\left(t_{i}, t_{N}\right) \xi_{t_{N}}-\prod_{k=i}^{N-1} \eta_{k}\left(t_{i}, t_{N}\right) \xi_{t_{N}}\right) d \mathbf{P}=0
\end{aligned}
$$

Доказательство леммы вытекает непосредственно из (6).

Пусть $A=\left\{0=t_{0} \leqslant t_{1} \leqslant \cdots \leqslant T\right\}$, обозначим

$$
\phi(s, t)=\prod_{k \geqslant 0} \zeta\left(t_{k}, t_{k+1}\right) I\left\{s \leqslant t_{k}<t_{k+1} \leqslant t\right\}, \quad 0 \leqslant s<t \leqslant T .
$$

Лемма 4. Пусть $\left\{\xi_{t_{n}}, \mathscr{F}_{t_{n}}, n \geqslant 0\right\}-$ - нотрицательный непрерывжый случайный прочесс, заданный на множестве $A$ и согласованный $c$ потоком $\left\{\mathscr{F}_{t_{n}}\right\}$. Предположим, что выполняются следующие условия:

1) $\mathbf{E} \sup _{n \geqslant 0} \xi_{t_{n}}<\infty$;

2) $0 \leqslant \sigma_{i} \leqslant T, i=1,2$, - моменты остановки относительно $\left\{\mathscr{F}_{t_{n}}, n \geqslant 0\right\}$, принимающие значения из множества $A$;

3) существует семейство $\zeta\left(t_{l}, t_{n}\right), 0 \leqslant t_{l} \leqslant t_{n}$, неотричательных $\mathscr{F}_{t_{l}}$-измеримых ограниченных в совокупности случайных величин таких, чmo

$$
\begin{aligned}
& \zeta\left(t_{l}, t_{n}\right) I\left\{\sigma_{1} \leqslant t_{l}<t_{n} \leqslant \sigma_{2}\right\} \leqslant 1, \\
& \zeta\left(t_{l}, t_{n}\right) \mathbf{E}\left(\xi_{t_{n}} \mid \mathscr{F}_{t_{l}}\right) I\left\{\sigma_{1}=t_{k} \leqslant t_{l}<t_{n} \leqslant \sigma_{2}\right\} \\
& \quad \geqslant \xi_{t_{l}} I\left\{\sigma_{1}=t_{k} \leqslant t_{l}<t_{n} \leqslant \sigma_{2}\right\} \quad \text { n.н., } \quad 0 \leqslant t_{k} \leqslant t_{l} \leqslant t_{n} \leqslant T .
\end{aligned}
$$

Tozda

$$
\mathbf{E}\left(\phi\left(\sigma_{1}, \sigma_{2}\right) \xi_{\sigma_{2}} \mid \mathscr{F}_{\sigma_{1}}\right) I\left\{\sigma_{1}<\sigma_{2}\right\} \geqslant \xi_{\sigma_{1}} I\left\{\sigma_{1}<\sigma_{2}\right\} \quad \text { n.н. }
$$

Д оказат ельст в о. Положим $\sigma_{i}(N)=\sigma_{i} \wedge t_{N}, N \geqslant 0, i=$ 1,2 . Тогда $\sigma_{i}(N)$ - дискретные моменты остановки относительно $\left\{\mathscr{F}_{t_{n}}\right.$, $n \geqslant 0\}$, и для $n \leqslant N$

$$
\begin{gathered}
\zeta\left(t_{l}, t_{n}\right) \mathbf{E}\left(\xi_{t_{n}} \mid \mathscr{F}_{t_{l}}\right) I\left\{\sigma_{1}(N)=t_{k} \leqslant t_{l}<t_{n} \leqslant \sigma_{2}(N)\right\} \\
\geqslant \xi_{t_{l}} I\left\{\sigma_{1}(N)=t_{k} \leqslant t_{l}<t_{n} \leqslant \sigma_{2}(N)\right\} \quad \text { п.н. }
\end{gathered}
$$

В силу (8) можно применить лемму 3. Переходя к пределу при $N \rightarrow \infty$, что возможно в силу условия 1), с использованием теоремы 1.6 [3, с. 25] получаем (7). Лемма доказана.

Пусть $\Lambda=\left\{\lambda_{n}, n \geqslant 1\right\}=\left\{0=t_{0}^{n}<t_{1}^{n}<\cdots<t_{k_{n}}^{n}=T, n \geqslant 1\right\}-$ некоторая последовательность разбиений отрезка $[0, T] ;\left\{\left(t_{k}^{n}, t_{k+1}^{n}\right)\right.$, $\left.1 \leqslant k \leqslant k_{n}-1, \quad n \geqslant 1\right\}$ - некоторое семейство случайньх величин, 
$\left\{\xi_{t}, \mathscr{F}_{t}, t \in[0, T]\right\}$ - положительный непрерывный случайный процесс. Введем обозначения:

$$
\begin{aligned}
\zeta(s, t) & =\frac{\xi_{s}}{\mathbf{E}\left(\xi_{t} \mid \mathscr{F}_{s}\right)}, \quad 0 \leqslant s \leqslant t \leqslant T, \\
\phi_{n}(s, t) & =\prod_{k=1}^{k_{n}-1} \zeta\left(t_{k}^{n}, t_{k+1}^{n}\right) I\left\{s \leqslant t_{k}^{n}<t_{k+1}^{n} \leqslant t\right\} .
\end{aligned}
$$

Лемма 5. Пусть $\left\{\xi_{t}, \mathscr{F}_{t}, t \in[0, T]\right\}-$ строго положительный непрерывный случайный прочесс, который удовлетворяет условию (2), $\theta_{2}-$ предсказуемый момент остановки такой, что для всех $0 \leqslant s \leqslant$ $t \leqslant T$

$$
\zeta(s, t) I\left\{s<t \leqslant \theta_{2}\right\}<1 \quad \text { n.н. }
$$

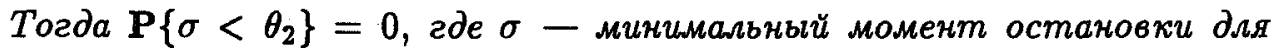
npoyecca $\xi_{t}$.

Д ок а з а те ль ст в о. Пусть $\sigma$ - минимальный момент остановки для $\xi_{t}$, положим

$$
\sigma_{m}=\left\{\begin{array}{l}
\frac{i}{2^{m}} T, \quad \frac{i-1}{2^{m}} T \leqslant \sigma<\frac{i}{2^{m}} T, \quad 1 \leqslant i<2^{m}, m \geqslant 1, \\
T, \quad \sigma=T .
\end{array}\right.
$$

Поскольку $\theta_{2}$ - предсказуемый момент остановки, то, согласно [4], сушествует последовательность $\left\{\tau_{m}, m \geqslant 1\right\}$ этажных моментов остановки, предвещающая $\theta_{2}$. Заметим, что $\mathbf{P}\left\{\sigma<\theta_{2}\right\}=\lim _{m \rightarrow \infty} \mathbf{P}\left\{\sigma_{m}<\tau_{m}\right\}$. Пусть $\left\{0 \leqslant t_{1}^{m}<t_{2}^{m}<\cdots\right\}-$ упорядоченное множество значений $\left\{\tau_{m}, \sigma_{m}\right\}$. Тогда имеют место соотношения

$$
\begin{aligned}
& \zeta\left(t_{l}^{m}, t_{n}^{m}\right) I\left\{\sigma_{m}<t_{l}^{m}<t_{n}^{m}<\tau_{m}\right\}<1, \\
& \zeta\left(t_{l}^{m}, t_{n}^{m}\right) \mathbf{E}\left(\xi_{t_{n}^{m}} \mid \mathscr{F}_{l}^{m}\right) I\left\{\sigma_{m}=t_{k}^{m}<t_{l}^{m}<t_{n}^{m} \leqslant \tau_{m}\right\} \\
& \quad=\xi_{t_{l}^{m}} I\left\{\sigma_{m}=t_{k}^{m}<t_{l}^{m}<t_{n}^{m} \leqslant \tau_{m}\right\} \quad \text { п.н., }
\end{aligned}
$$

т.е. выполняется условие 3) леммы 4 для семейства случайных величин $\zeta(s, t)$, определенных соотношением (9). Поэтому в силу (10) и леммы 4 имеем:

$$
\mathbf{E}\left(\phi_{m}\left(\sigma_{m}, \tau_{m}\right) \xi_{\tau_{m}} I\left\{\sigma_{m}<\tau_{m}\right\} \mid \mathscr{F}_{\sigma_{m}}\right) \geqslant \xi_{\sigma_{m}} I\left\{\sigma_{m}<\tau_{m}\right\} \quad \text { п.н. }
$$

Очевидно, тогда

$$
\mathbf{P}\left\{\sigma_{m}<\tau_{m}\right\} \leqslant \mathbf{P}\left\{\mathbf{E}\left(\phi_{m}\left(\sigma_{m}, \tau_{m}\right) \xi_{\tau_{m}} \mid \mathscr{F}_{\sigma_{m}}\right) \geqslant \xi_{\sigma_{m}}\right\} .
$$

Пусть $m \rightarrow \infty$, тогда $\xi_{\sigma_{m}} \rightarrow \xi_{\sigma}, \xi_{\tau_{m}} \rightarrow \xi_{\theta_{2}}$, и в силу ,(11) $\varlimsup_{m \rightarrow \infty} \phi_{m}\left(\sigma_{m}, \tau_{m}\right)<1$. Следовательно, $\mathbf{P}\left\{\sigma<\theta_{2}\right\} \leqslant \mathbf{P}\left\{\mathbf{E}\left(\xi_{\theta_{2}} \mid \mathscr{F}_{\sigma}\right)>\right.$ 
$\left.\xi_{\sigma}\right\}=0$, поскольку, согласно [2], при выполнении условия (2) имеет место равенство $f_{\sigma}=\xi_{\sigma}$. Лемма доказана.

Пусть теперь $\left\{\xi_{t}, \mathscr{F}_{t}, t \in[0, T]\right\}-$ положительный п.н. непрерывный случайный процесс, для которого выполняется условие (2).

$\mathrm{O}$ п р е д е л е н и е 2 . Предсказуемый момент остановки $\theta \in[0, T]$ будем называть переломным для процесса $\xi_{t}$, если $\theta$ удовлетворяет неравенствам (3) и (11), т.е. $\theta=\theta_{1}=\theta_{2}$ п.н.

Термин «переломный момент остановки» можно объяснить тем, что до этого момента процесс $\xi_{t}$ имеет свойства, сходные с субмартингальными, а после него - свойства типа супермартингальных.

Непосредственно из лемм 2 и 5 вытекает

Теорема 1. Пусть $\left\{\xi_{t}, \mathscr{F}_{t}, t \in[0, T]\right\}-$ положительный жепрерывный прочесс, который удовлетворяет условию (2). Если для прочесса $\xi_{t}$ существует переломный момент остановки, то этот момент совпадает с минимальным, а следовательно, является оптимальным.

3 а м е ч а н и е 1. В статье [5] приведен контрпример, показывающий, что огибаюшая Снелла не всегда совпадает с исходным процессом после минимального, в наших терминах, момента остановки. Теорема 1 фактически содержит одно из достаточных условий такого совпадения.

3. Отыскание оптимального момента переключения. Пусть $a_{i}(s), b_{i}(s), s \in[0, T]$, суть $\left\{\mathscr{F}_{s}\right\}$-согласованные случайные функции, $a_{i}(s) \geqslant 0, \mathbf{E} \int_{0}^{T} a_{i}(s) d s<\infty, \mathbf{E} \int_{0}^{T} b_{i}^{2}(s) d s<\infty, i=1,2$. Не теряя общности, будем считать, что $a_{1}(0)>a_{2}(0)$ и что в момент $t=0$ инвестор начинает формировать свой портфель, которому соответствует коэффициент роста $a_{1}(s)$ и коэффициент волатильности $b_{1}(s)$. Пусть начальный капитал инвестора равен $X_{0}$. Для упрощения, не ограничивая общности, можем считать, что $X_{0}=1$ с вероятностью 1 . Капитал инвестора описывается линейным стохастическим дифференциальным уравнением

$$
\tilde{X}_{t}=1+\int_{0}^{t} a_{1}(s) \tilde{X}_{s} d s+\int_{0}^{t} b_{1}(s) \tilde{X}_{s} d w_{s}, \quad t \in[0, T]
$$

где $\left\{w_{t}, \mathscr{F}_{t}, t \in[0, T]\right\}-$ винеровский процесс. Пусть $\tau \in[0, T]-$ некоторый момент остановки, выбранный инвестором. В этот момент он забирает все средства с указанного первого счета, уплачивая при этом некоторую цену $C(\tau)$. После этого он распределяет оставшийся капитал $\tilde{X}_{\tau}-C(\tau)$ между облигациями и акциями, которым соответствуют коэффициент роста $a_{2}(s)$ и коэффициент волатильности $b_{2}(s)$. В зависимости от вида уплачиваемой цены $C(\tau)$ рассмотрим два случая.

1. Це на пе реключения пропорпиональна к а п и т а л у. Пусть $C(\tau)=c_{\tau} \widetilde{X}_{\tau}$, где $c_{t}$ есть $\mathscr{F}_{t}$-измеримая непрерывная случайная функция, $\mathbf{P}\left\{0<c_{t}<1, t \in[0, T]\right\}=1$. По предположению, после момента переключения $\tau$ капитал инвестора описывается 
линейным стохастическим дифференциальным уравнением

$$
X_{t}=\tilde{X}_{\tau}\left(1-c_{\tau}\right)+\int_{\tau}^{t} a_{2}(s) X_{s} d s+\int_{\tau}^{t} b_{2}(s) X_{s} d w_{s}, \quad t \in[\tau, T] .
$$

Уравнения (12) и (13) имеют, соответственно, решения вида

$$
\begin{gathered}
\tilde{X}_{t}=\exp \left\{\int_{0}^{t}\left(a_{1}(s)-\frac{b_{1}^{2}(s)}{2}\right) d s+\int_{0}^{t} b_{1}(s) d w_{s}\right\}, \quad t \in[0, \tau] \\
X_{t}=\tilde{X}_{\tau}\left(1-c_{\tau}\right) \exp \left\{\int_{\tau}^{t}\left(a_{2}(s)-\frac{b_{2}^{2}(s)}{2}\right) d s\right. \\
\left.+\int_{\tau}^{t} b_{2}(s) d w_{s}\right\}, \quad t \in[\tau, T]
\end{gathered}
$$

Будем считать, что инвестор в любом случае забирает средства с первого счета и уплачивает цену, даже если $\tau=T$, но не забирает средства со второго счета. В этом случае, с учетом (15), задачей инвестора является максимизация величины

$$
\mathbf{E} X_{T}=\mathbf{E} \tilde{X}_{\tau}\left(1-c_{\tau}\right) \exp \left\{\int_{\tau}^{T}\left(a_{2}(s)-\frac{b_{2}^{2}(s)}{2}\right) d s+\int_{\tau}^{T} b_{2}(s) d w_{s}\right\}
$$

где $\tilde{X}_{\tau}$ определяется из (14) при $t=\tau$.

Лемма 6. Пусть выполняются следующие условия:

1) случайный прочесс $\left\{a_{2}(t), t \in[0, T]\right\}$ не зависит от совокупности прочессов $\left\{b_{2}(t), w_{t}, t \in[0, T]\right\}$

2) $\mathbf{E} \exp \left\{4 \int_{0}^{T} a_{i}(s) d s+6 \int_{0}^{T} b_{i}^{2}(s) d s\right\}<\infty, i=1,2$;

3) $\mathbf{E} \exp \left\{8 \int_{0}^{T} b_{i}^{2}(s) d s\right\}<\infty, i=1,2$.

Tozda

$$
\mathbf{E} X_{T}=\mathbf{E} \tilde{X}_{\tau}\left(1-c_{\tau}\right) \exp \left\{\int_{\tau}^{T} a_{2}(s) d s\right\} .
$$

Доказ ат тл вство. Обозначим $\tilde{a}(s)=a_{1}(s) I\{s \leqslant \tau\}+$ $a_{2}(s) I\{s>\tau\}, \widetilde{b}(s)=b_{1}(s) I\{s \leqslant \tau\}+b_{2}(s) I\{s \geq \tau\}$. Тогда в силу [6] и условия 3) процесс $\eta_{t}=\exp \left\{\int_{0}^{t} 4 \widetilde{b}(s) d w_{s}-\int_{0}^{t} 8 \widetilde{b}^{2}(s) d s\right\}$ является мартингалом. Кроме того, $\eta_{0}=1$, следовательно, $\mathbf{E} \eta_{T}=1$. Отсюда и из условия 2) вытекает, что

$$
\begin{aligned}
\mathbf{E} X_{T}^{2} \leqslant & \left(\mathbf{E} \exp \left\{\int_{0}^{T}\left(4 \tilde{a}(s)+6 \tilde{b}^{2}(s)\right) d s\right\}\right. \\
& \left.\times \mathbf{E} \exp \left\{\int_{0}^{T} 4 \widetilde{b}(s) d w_{s}-\int_{0}^{T} 8 \widetilde{b}^{2}(s) d s\right\}\right)^{1 / 2}<\infty
\end{aligned}
$$

а также и конечность правой части (16). 
Заметим, что, полагая $\tilde{a}(s)=a_{2}(s) I\{s>\tau\}, \tilde{b}(s)=b_{2}(s) \times$ $I\{s>\tau\}$, аналогично можно доказать, что $\mathbf{E} \exp \left\{\int_{\tau}^{T}\left(a_{2}(s)-b_{2}^{2}(s) / 2\right) d s+\right.$ $\left.\int_{\tau}^{T} b_{2}(s) d w_{s}\right\}<\infty$, в частности, для любого $t \in[0, T]$

$$
\mathbf{E} \exp \left\{\int_{t}^{T}\left(a_{2}(s)-\frac{b_{2}^{2}(s)}{2}\right) d s+\int_{t}^{T} b_{2}(s) d w_{s}\right\}<\infty
$$

Для доказательства леммы покажем сначала, что для любого момента остановки $\tau \in[0, T]$

$$
\begin{aligned}
& \mathbf{E}\left(\exp \left\{\int_{\tau}^{T}\left(a_{2}(s)-\frac{b_{2}^{2}(s)}{2}\right) d s+\int_{\tau}^{T} b_{2}(s) d w_{s}\right\} \mid \mathscr{F}_{\tau}\right) \\
& =\mathbf{E}\left(\exp \left\{\int_{\tau}^{T} a_{2}(s) d s\right\} \mid \mathscr{F}_{\tau}\right) .
\end{aligned}
$$

Пусть сначала $\tau$ - дискретный момент остановки, принимающий значения $0 \leqslant t_{1} \leqslant t_{2} \leqslant \cdots \leqslant t_{n}=T$. Тогда для любого $k=1, \ldots, n$

$$
\begin{gathered}
\mathbf{E}\left(\exp \left\{\int_{\tau}^{T}\left(a_{2}(s)-\frac{b_{2}^{2}(s)}{2}\right) d s+\int_{\tau}^{T} b_{2}(s) d w_{s}\right\} \mid \mathscr{F}_{\tau}\right) I\left\{\tau=t_{k}\right\} \\
=\mathbf{E}\left(\operatorname { e x p } \left\{\int_{t_{k}}^{T}\left(a_{2}(s)-\frac{b_{2}^{2}(s)}{2}\right) d s\right.\right. \\
\left.\left.+\int_{t_{k}}^{T} b_{2}(s) d w_{s}\right\} \mid \mathscr{F}_{t_{k}}\right) I\left\{\tau=t_{k}\right\} \quad \text { п.н. }
\end{gathered}
$$

Обозначим $\mathscr{F}_{a_{2}}=\sigma\left\{a_{2}(s), s \in[0, T]\right\}, \widehat{\mathscr{F}}_{t}=\mathscr{F}_{t} \vee \mathscr{F}_{a_{2}} \cdot$ Тогда

$$
\begin{aligned}
& \mathbf{E}\left(\exp \left\{\int_{t_{k}}^{T}\left(a_{2}(s)-\frac{b_{2}^{2}(s)}{2}\right) d s+\int_{t_{k}}^{T} b_{2}(s) d w_{s}\right\} \mid \mathscr{F}_{t_{k}}\right) \\
& =\mathbf{E}\left(\left(\exp \left\{\int_{t_{k}}^{T}\left(a_{2}(s)-\frac{b_{2}^{2}(s)}{2}\right) d s+\int_{t_{k}}^{T} b_{2}(s) d w_{s}\right\} \mid \widehat{\mathscr{F}}_{t_{k}}\right) \mid \mathscr{F}_{t_{k}}\right) .
\end{aligned}
$$

Обозначим $\widehat{M}_{t, s}=\exp \left\{-\int_{t}^{s}\left(b_{2}^{2}(u) / 2\right) d u+\int_{t}^{s} b_{2}(u) d w_{u}\right\}, s \geqslant t \geqslant 0$. Очевидно, $\mathbf{E} \widehat{M}_{t, T}<\infty$ в силу (18),

$$
\begin{aligned}
\mathbf{E} \widehat{M}_{t, T}^{2}=\mathbf{E} & \exp \left\{-\int_{t}^{T} b_{2}^{2}(s) d s+2 \int_{t}^{T} b_{2}(s) d w_{s}\right\} \\
\times & \left(\mathbf{E} \exp \left\{-8 \int_{t}^{T} b_{2}^{2}(s) d s+4 \int_{t}^{T} b_{2}(s) d w_{s}\right\}\right. \\
& \left.\times \mathbf{E} \exp \left\{6 \int_{t}^{T} b_{2}^{2}(s) d s\right\}\right)^{1 / 2} .
\end{aligned}
$$


Случайный процесс $\tilde{\eta}_{s}=\exp \left\{4 \int_{t}^{s} b_{2}(u) d w_{u}-8 \int_{t}^{s} b_{2}^{2}(u) d u\right\}, s \in[t, T]$, является мартингалом в силу условия 3) и $\tilde{\eta}_{t}=1$. Поэтому $\mathbf{E} \tilde{\eta}_{T}=1$, а тогда $\mathbf{E} \widehat{M}_{t, T}^{2} \leqslant\left(\mathbf{E} \exp \left\{6 \int_{t}^{T} b_{2}^{2}(s) d s\right\}\right)^{1 / 2}<\infty$ в силу условия 2$)$. Далее, $\widehat{M}_{t, T}=1+\int_{t}^{T} b_{2}(s) \widehat{M}_{t, s} d w_{s}$, и при этом

$$
\mathbf{E} \int_{t}^{T} b_{2}^{2}(s) \widehat{M}_{t, s}^{2} d s=\mathbf{E}\left(\int_{t}^{T} b_{2}(s) \widehat{M}_{t, s} d w_{s}\right)^{2}=\mathbf{E}\left(\widehat{M}_{t, T}-1\right)^{2}<\infty .
$$

Поэтому

$$
\mathbf{E}\left(\widehat{M}_{t_{k}, T} \mid \widehat{\mathscr{F}}_{t_{k}}\right)=1+\mathbf{E}\left(\int_{t_{k}}^{T} b_{2}(s) \widehat{M}_{t_{k}, s} d w_{s} \mid \widehat{\mathscr{F}}_{t_{k}}\right) .
$$

Пусть $\lambda_{n}=\left\{t_{k}=s_{1}^{n}<\cdots<s_{n}^{n}=T\right\}-$ последовательность разбиений отрезка $\left[t_{k}, T\right]$, которые измельчаются. Тогда

$$
\begin{aligned}
& \mathbf{E}\left(\int_{t_{k}}^{T} b_{2}(s) \widehat{M}_{t_{k}, s} d w_{s} \mid \widehat{\mathscr{F}}_{t_{k}}\right)=\lim _{\left|\lambda_{n}\right| \rightarrow 0} \mathbf{E}\left(\sum_{i} b_{2}\left(s_{i}^{n}\right) \widehat{M}_{t_{k}, s_{i}^{n}} \Delta w_{s_{i}^{n}} \mid \widehat{\mathscr{F}}_{t_{k}}\right) \\
& \quad=\lim _{\left|\lambda_{n}\right| \rightarrow 0} \mathbf{E}\left(\sum_{i} b_{2}\left(s_{i}^{n}\right) \widehat{M}_{t_{k}, s_{i}^{n}} \mathbf{E}\left(\Delta w_{s_{i}^{n}} \mid \widehat{\mathscr{F}}_{s_{i}^{n}}\right) \mid \widehat{\mathscr{F}}_{t_{k}}\right) .
\end{aligned}
$$

Поскольку $\Delta w_{s_{i}^{n}}=w_{s_{i+1}^{n}}-w_{s_{i}^{n}}$ не зависит от $\widehat{\mathscr{F}}_{s_{i}^{n}}$ в силу условия 1 ), то $\mathbf{E}\left(\Delta w_{s_{i}^{n}} \mid \widehat{\mathscr{F}}_{s_{i}^{n}}\right)=\mathbf{E} \Delta w_{s_{i}^{n}}=0$. Следовательно, $\mathbf{E}\left(\widehat{M}_{t_{k}}, T \mid \widehat{\mathscr{F}}_{t_{k}}\right)=1$. Поэтому правая часть (21) равна $\mathrm{E}\left(\exp \left\{\int_{t_{k}}^{T} a_{2}(s) d s\right\} \mid \mathscr{F}_{t_{k}}\right)$, а тогда, учитывая (20), получаем (19) для любого дискретного момента остановки.

Пусть теперь $\tau$ - произвольный момент остановки, положим

$$
\tau_{n}=\sum_{k=0}^{2^{n}-1} \frac{k T}{2^{n}} I\left\{\frac{(k-1) T}{2^{n}} \leqslant \tau<\frac{k T}{2^{n}}\right\}+T I\{\tau=T\}, \quad n \geqslant 1 .
$$

Тогда (19) имеет место для $\tau_{n}, n \geqslant 1$, откуда

$$
\begin{aligned}
& \mathbf{E} \tilde{X}_{\tau_{n}}\left(1-c_{\tau_{n}}\right) \exp \left\{\int_{\tau_{n}}^{T}\left(a_{2}(s)-\frac{b_{2}^{2}(s)}{2}\right) d s+\int_{\tau_{n}}^{T} b_{2}(s) d w_{s}\right\} \\
& =\mathbf{E} \tilde{X}_{\tau_{n}}\left(1-c_{\tau_{n}}\right) \exp \left\{\int_{\tau_{n}}^{T} a_{2}(s) d s\right\} .
\end{aligned}
$$

Если $n \rightarrow \infty$, то случайные величины под знаком математического ожидания в левой и правой частях (22) стремятся к соответствующим значениям при $\tau$ вместо $\tau_{n}$. Равномерная интегрируемость вытекает из оценки (17). Лемма доказана.

Рассмотрим теперь $\left\{\mathscr{F}_{t}\right\}$-согласованный случайный процесс

$$
Y_{t}=\tilde{X}_{t}\left(1-c_{t}\right) \mathbf{E}\left(\exp \left\{\int_{t}^{T} a_{2}(s) d s\right\} \mid \mathscr{F}_{t}\right)
$$


В силу леммы $6 \mathbf{E} Y_{T}=\mathbf{E} X_{T}, \mathbf{n}$, таким образом, задача состоит в нахождении $\sup _{\tau \in[0, T]} \mathbf{E} Y_{\tau}$. Согласно теореме 1, $\sup _{\tau \in[0, T]} \mathbf{E} Y_{\tau}=\mathbf{E} Y_{\theta}$, где $\theta-$ переломный момент остановки, если он существует. В следующей теореме сформулируем условия, достаточные для существования переломного момента остановки для процесса $Y_{t}$. Для этого введем в рассмотрение случайную функцию

$$
\psi(t)=\left(1-c_{t}\right) \exp \left\{\int_{0}^{t}\left(a_{1}(s)-a_{2}(s)\right) d s\right\}, \quad t \in[0, T] .
$$

Теорема 2. Пусть выполняются условия леммы 6, а тахже следуюшие условия:

1) случайжые прочессы $\left\{a_{1}(t), a_{2}(t), c_{t}, t \in[0, T]\right\}$ не зависят в совокупности от случайных прочессов $\left\{b_{1}(t), w_{t}, t \in[0, T]\right\}$;

2) $\int_{0}^{T} a_{i}(t) d t \quad \mathscr{F}_{0}$-измеримы, $i=1,2$;

3) существует предсказуемый момент остановки $\theta$ такой, что $\mathbf{E}\left(\psi(t) \mid \mathscr{F}_{s}\right) I\{s<t \leqslant \theta\}>\psi(s) I\{s<t \leqslant \theta\} u \psi(t)$ yбывает на $[\theta, T]$.

Тогда $\theta$ - переломный момент остановки для прочесса $Y_{t}$.

Д ок аз ат ельст в о. Заметим, что $Y_{t}>0$ для всех $t \in[0, T]$ с вероятностью 1. Рассмотрим для всех $0 \leqslant s \leqslant t \leqslant T$

$$
\begin{aligned}
\mathbf{E}\left(Y_{t} \mid \mathscr{F}_{s}\right)= & \mathbf{E}\left(\tilde{X}_{t}\left(1-c_{t}\right) \exp \left\{\int_{t}^{T} a_{2}(u) d u\right\} \mid \mathscr{F}_{s}\right) \\
= & \tilde{X}_{s} \mathbf{E}\left(( 1 - c _ { t } ) \operatorname { e x p } \left\{\int_{s}^{t}\left(a_{1}(u)-\frac{b_{1}^{2}(u)}{2}\right) d u+\int_{s}^{t} b_{1}(u) d w_{u}\right.\right. \\
& \left.\left.+\int_{t}^{T} a_{2}(u) d u\right\} \mid \mathscr{F}_{s}\right) .
\end{aligned}
$$

При выполнении условия 1) теоремы аналогично лемме 6 можно доказать, что условное математическое ожидание в правой части последнего равенства равно $\mathbf{E}\left(\left(1-c_{t}\right) \exp \left\{\int_{s}^{t} a_{1}(u) d u+\int_{t}^{T} a_{2}(u) d u\right\} \mid \mathscr{F}_{s}\right)$. Поэтому, с учетом условия 2),

$$
\mathbf{E}\left(Y_{t} \mid \mathscr{F}_{s}\right)=\frac{Y_{s}}{1-c_{s}} \mathbf{E}\left(\left(1-c_{t}\right) \exp \left\{\int_{s}^{t}\left(a_{1}(u)-a_{2}(u)\right) d u\right\} \mid \mathscr{F}_{s}\right) .
$$

Покажем теперь, что $\mathbf{E} \sup _{t \in[0, T]} Y_{t}<\infty$. Действительно,

$$
\mathbf{E} \sup _{t \in[0, T]} Y_{t} \leqslant\left(\mathbf{E} \sup _{t \in[0, T]} \widetilde{X}_{t}^{2} \cdot \mathbf{E} \sup _{t \in[0, T]}\left[\mathbf{E}\left(\exp \left\{\int_{t}^{T} a_{2}(u) d u\right\} \mid \mathscr{F}_{t}\right)\right]^{2}\right)^{1 / 2}
$$

Опеним каждый из сомножителей правой части последнего неравенства:

$$
\mathbf{E} \sup _{t \in[0, T]} \tilde{X}_{t}^{2} \leqslant \mathbf{E} \sup _{t \in[0, T]} \exp \left\{\int_{0}^{t}\left(2 a_{1}(s)-b_{1}^{2}(s)\right) d s+\int_{0}^{t} 2 b_{1}(s) d w_{s}\right\}
$$




$$
\begin{aligned}
& \leqslant\left(\mathbf{E} \sup _{t \in[0, T]} \exp \left\{\int_{0}^{t}\left(4 a_{1}(s)+2 b_{1}^{2}(s)\right) d s\right\}\right. \\
& \left.\quad \times \mathbf{E} \sup _{t \in[0, T]} \exp \left\{\int_{0}^{t} 4 b_{1}(s) d w_{s}-\int_{0}^{t} 4 b_{1}^{2}(s) d s\right\}\right)^{1 / 2}
\end{aligned}
$$

Поскольку, согласно [6], процесс $\exp \left\{\int_{0}^{t} 2 b_{1}(s) d w_{s}-\int_{0}^{t} 2 b_{1}^{2}(s) d s\right\}-$ мартингал, то по неравенству Дуба

$$
\begin{aligned}
& \mathbf{E} \sup _{t \in[0, T]} \exp \left\{\int_{0}^{t} 4 b_{1}(s) d w_{s}-\int_{0}^{t} 4 b_{1}^{2}(s) d s\right\} \\
& \leqslant 4 \mathbf{E} \exp \left\{\int_{0}^{T} 4 b_{1}(s) d w_{s}-\int_{0}^{T} 4 b_{1}^{2}(s) d s\right\}<\infty
\end{aligned}
$$

в силу условия 2) леммы 6, и, следовательно, с учетом этого же условия $\operatorname{Esup}_{t \in[0, T]} \widetilde{X}_{t}^{2}<\infty$. Далее, снова в силу неравенства Дуба

$$
\mathbf{E} \sup _{t \in[0, T]}\left[\mathbf{E}\left(\exp \left\{\int_{t}^{T} a_{2}(u) d u\right\} \mid \mathscr{F}_{t}\right)\right]^{2} \leqslant 4 \mathbf{E} \exp \left\{2 \int_{0}^{T} a_{2}(u) d u\right\}<\infty .
$$

Таким образом, мы показали, что $\mathbf{E} \sup _{t \in[0, T]} Y_{t}<\infty$.

Нетрудно проверить, что в силу условия 3) и равенства (23) для $\theta$ и процесса $Y_{t}$ выполняются неравенства (3) и (11). Таким образом, $\theta$ является переломным моментом остановки для процесса $Y_{t}$, и теорема доказана.

П р и м е р 1. Пусть $c_{t}$ - дифференцируемая функция, функция $g_{t}=a_{1}(t)-a_{2}(t)-c_{t}^{\prime} /\left(1-c_{t}\right)$ непрерывна. Если сушествует предсказуемый момент остановки $\theta$ такой, что $\mathbf{E}\left(\exp \left\{\int_{s}^{t}\left(a_{1}(u)-a_{2}(u)\right) d u\right\}\left(1-c_{t}\right) g_{t}\right.$ $\left.\mathscr{F}_{s}\right) I\{s<t \leqslant \theta\}>0$ и $g_{t} I\{t \geqslant \theta\} \leqslant 0$, то момент остановки $\theta$ является

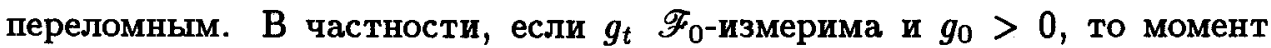
остановки $\theta=\inf \left\{t: g_{t}=0\right\}$ является переломным.

2. Ц е а пе реклю чения с о дежит компоненту, не з а и ся у о от капи т ала. Пусть $C_{\tau}=c_{\tau} \widetilde{X}_{\tau}+\gamma_{\tau}$, где $c_{t}$ - дифференцируемая неслучайная функция, $0<c_{t}<1, \gamma_{t}=\gamma e^{-\beta t}$, $0<\gamma \leqslant 1, \beta>0$ - некоторые неслучайные постоянные, $a_{1}(s), a_{2}(s)-$ непрерывные неслучайные функции, $a_{1}(0)>a_{2}(0) \geqslant 0$. Как и прежде, после переключения капитал инвестора описывается линейным стохастическим дифференциальньм уравнением

$$
X_{t}=\tilde{X}_{\tau}\left(1-c_{\tau}\right)-\gamma e^{-\beta t}+\int_{\tau}^{t} a_{2}(s) X_{s} d s+\int_{\tau}^{t} b_{2}(s) X_{s} d w_{s}, \quad t \in[\tau, T]
$$

где $\tilde{X}_{t}$ определяется из (14). В зависимости от условий договора рассмотрим два случая. 
(a) Будем считать, что инвестор уплачивает цену в момент переключения $\tau$, даже если $\tau=T$, и забирает средства с первого счета, но не забирает со второго. Отрицательный капитал будет означать взятие денег в долг.

Аналогично предыдущему, нетрудно показать, что задача максимизации $\mathbf{E} X_{T}$ сводится к отысканию оптимального момента остановки для процесса

$$
Y_{t}=\left(\tilde{X}_{t}\left(1-c_{t}\right)-\gamma e^{-\beta t}\right) \exp \left\{\int_{t}^{T} a_{2}(s) d s\right\} .
$$

Введем некоторые обозначения:

$$
\begin{aligned}
M_{t} & =\exp \left\{\int_{0}^{t} b_{1}(s) d w_{s}-\int_{0}^{t} \frac{b_{1}^{2}(s)}{2} d s\right\}, \quad t \geqslant 0, \\
A_{t} & =\exp \left\{\int_{0}^{t}\left(a_{1}(s)-a_{2}(s)\right) d s\right\}, \quad 0 \leqslant t \leqslant T, \\
a_{t} & =A_{t}\left[\left(1-c_{t}\right)\left(a_{1}(t)-a_{2}(t)\right)-c_{t}^{\prime}\right], \quad 0 \leqslant t \leqslant T, \\
b_{t} & =\gamma \exp \left\{-\int_{0}^{t}\left(a_{2}(s)+\beta\right) d s\right\}\left(a_{2}(t)+\beta\right), \quad 0 \leqslant t \leqslant T,
\end{aligned}
$$

и будем считать, что $A_{t}=A_{T}, a_{t}=a_{T}, b_{t}=b_{T}, b_{1}(t)=b_{1}(T), t \geqslant T$.

Теорема 3. Пусть выполняются условия:

1) функция $a_{2}(t)-a_{1}(t)$ строго возрастает по $t \in[0, T]$;

2) $c_{t}^{\prime}<0$ длs всеx $t \in[0, T] u$ возрастаem no $t \in[0, T]$.

Тогда момент остановки

$$
\sigma_{0}=\inf \left\{t>0: M_{t} a_{t}+b_{t}=0\right\} \wedge T
$$

является оптимальным моментом остановки для прочесса $Y_{t}$.

Д ок а а т е л с т в о. Заметим, что процесс $M_{t}$ является мартингалом, $M_{0}=1$, и по формуле Ито

$$
M_{t}=1+\int_{0}^{t} b_{1}(s) M_{s} d w_{s}
$$

Рассмотрим процесс $\widehat{X}_{t}=A_{t} M_{t}$. Используя формулу Ито, нетрудно получить равенства:

$$
\begin{aligned}
& \widehat{X}_{t}=1+\int_{0}^{t} A_{s} d M_{s}+\int_{0}^{t} M_{s} A_{s}\left(a_{1}(s)-a_{2}(s)\right) d s \\
& \widehat{X}_{t}\left(1-c_{t}\right)=1-c_{0}+\int_{0}^{t}\left(1-c_{s}\right) A_{s} d M_{s}+\int_{0}^{t} M_{s} a_{s} d s \\
&-\gamma e^{-\beta t} \exp \left\{-\int_{0}^{t} a_{2}(s) d s\right\}=-\gamma+\int_{0}^{t} b_{s} d s
\end{aligned}
$$


Тогда в силу (14)

$$
\tilde{X}_{t}\left(1-c_{t}\right) \exp \left\{-\int_{0}^{t} a_{2}(s) d s\right\}=A_{t} M_{t}\left(1-c_{t}\right)=\hat{X}_{t}\left(1-c_{t}\right) .
$$

Теперь перепишем (24) с учетом (25)-(27):

$Y_{t}=\exp \left\{\int_{0}^{T} a_{2}(s) d s\right\}\left[1-c_{0}-\gamma+\int_{0}^{t}\left(1-c_{s}\right) A_{s} d M_{s}+\int_{0}^{t}\left(M_{s} a_{s}+b_{s}\right) d s\right]$.

Поскольку $\int_{0}^{t}\left(1-c_{s}\right) A_{s} d M_{s}$ является мартингалом и для любого момента остановки $\tau \in[0, T]$ выполнено равенство $\mathbf{E} \int_{0}^{\tau}\left(1-c_{s}\right) A_{s} d M_{s}=0$, то, таким образом, задача нахождения оптимального момента остановки для процесса $Y_{t}$ сводится к отысканию момента остановки, на котором достигается $S:=\sup _{\tau \in[0, T]} \mathbf{E} \int_{0}^{\tau}\left(M_{s} a_{s}+b_{s}\right) d s$.

Заметим, что

$$
M_{0} a_{0}+b_{0}=\left(1-c_{0}\right)\left(a_{1}(0)-a_{2}(0)\right)-c_{0}^{\prime}+\gamma\left(a_{2}(0)+\beta\right)>0 .
$$

Обозначим $\tau_{0}=\inf \left\{t>0: M_{t} a_{t}+b_{t}=0\right\}$ и покажем, что момент остановки $\sigma_{0}=\tau_{0} \wedge T$ является оптимальным, т.е. $S=\mathrm{E} \int_{0}^{\sigma_{0}}\left(M_{s} a_{s}+b_{s}\right) d s$.

Покажем сначала, что любой момент остановки $\tau<\sigma_{0}$ не является оптимальным. Действительно, пусть $\tau \leqslant T-$ произвольный момент остановки. Рассмотрим множество $A_{0}=\left\{\tau<\sigma_{0}\right\} \subset \Omega$, и пусть $\mathbf{P}\left(A_{0}\right) \neq 0$. Положим $\hat{\tau}=\tau I_{\bar{A}_{0}}+\sigma_{0} I_{A_{0}}$. Тогда для любого $u \leqslant T$ имеем: $\{\hat{\tau} \leqslant u\}=\{\tau \leqslant u\} \cap\left\{\omega \in \bar{A}_{0}\right\}+\left\{\sigma_{0} \leqslant u\right\} \cap\left\{\omega \in A_{0}\right\}$. Заметим, что $\left\{\tau<\sigma_{0}\right\}=\left\{\omega \in A_{0}\right\} \in \mathscr{F}_{\tau},\{\tau \leqslant u\} \cap\left\{\omega \in \bar{A}_{0}\right\} \in \mathscr{F}_{u}$, $\left\{\sigma_{0} \leqslant u\right\} \cap\left\{\omega \in A_{0}\right\} \in \mathscr{F}_{u}$. Значит, $\widehat{\tau}$ - момент остановки относительно $\left\{\mathscr{F}_{t}, t \leqslant T\right\}$. Теперь

$$
\begin{aligned}
\mathbf{E} \int_{0}^{\hat{\tau}}\left(M_{s} a_{s}+b_{s}\right) d s & =\mathbf{E} \int_{0}^{\tau}\left(M_{s} a_{s}+b_{s}\right) d s I_{\bar{A}_{0}}+\mathbf{E} \int_{0}^{\sigma_{0}}\left(M_{s} a_{s}+b_{s}\right) d s I_{A_{0}} \\
& >\mathbf{E} \int_{0}^{\tau}\left(M_{s} a_{s}+b_{s}\right) d s,
\end{aligned}
$$

т.е. $\tau$ - не оптимальный момент остановки. Таким образом, $S=$ $\sup _{\tau \geqslant \sigma_{0}} \mathbf{E} \int_{0}^{\tau}\left(M_{s} a_{s}+b_{s}\right) d s$.

Покажем теперь, что для любого момента остановки $\tau \geqslant \sigma_{0}$

$$
\mathbf{E} \int_{0}^{\tau}\left(M_{s} a_{s}+b_{s}\right) d s \leqslant \mathbf{E} \int_{0}^{\sigma_{0}}\left(M_{s} a_{s}+b_{s}\right) d s .
$$

Заметим сначала, что если $a_{t} \geqslant 0$ на $[0, T]$, то $\sigma_{0}=T$. Предположим, что существует $t_{0}<T$ такое, что $a_{t_{0}}=0$. Тогда, очевидно, $a_{t}>0$ при $t \leqslant t_{0}$ и $a_{t}<0$ при $t>t_{0}$. Очевидно также, что $\sigma_{0}>t_{0}$. Рассмотрим отношение

$$
-\frac{b_{t}}{a_{t}}=\frac{e^{-\beta t}\left(a_{2}(t)+\beta\right)}{\exp \left\{\int_{0}^{t} a_{1}(s) d s\right\}\left[\left(1-c_{t}\right)\left(a_{2}(t)-a_{1}(t)\right)+c_{t}^{\prime}\right]} .
$$


Поскольку $-b_{t} / a_{t}$ убывает по $t$, то для любого $t$ такого, что $\sigma_{0}<t<T$, имеет место неравенство $-b_{\sigma_{0}} / a_{\sigma_{0}} \geqslant-b_{t} / a_{t}$. Рассмотрим случайный процесс $\zeta_{s}=\mathbf{E}\left(\int_{\sigma_{0} \wedge s}^{s}\left(M_{u} a_{u}+b_{u}\right) d u \mid \mathscr{F}_{s \wedge \sigma_{0}}\right), 0 \leqslant s \leqslant T$. Для любых $0 \leqslant s<t \leqslant T$

$$
\begin{aligned}
\mathbf{E}\left(\zeta_{t}-\zeta_{s} \mid \mathscr{F}_{s \wedge \sigma_{0}}\right)=\mathbf{E}( & \int_{\sigma_{0}}^{t}\left(M_{u} a_{u}+b_{u}\right) d u I\left\{s \leqslant \sigma_{0}<t\right\} \\
& \left.+\int_{s}^{t}\left(M_{u} a_{u}+b_{u}\right) d u I\left\{\sigma_{0}<s\right\} \mid \mathscr{F}_{s \wedge \sigma_{0}}\right) .
\end{aligned}
$$

Ho

$$
\begin{aligned}
& \mathbf{E}\left(\int_{\sigma_{0}}^{t}\left(M_{u} a_{u}+b_{u}\right) d u I\left\{s \leqslant \sigma_{0}<t\right\} \mid \mathscr{F}_{s \wedge \sigma_{0}}\right) \\
& =\mathbf{E}\left(\int_{s}^{t}\left(M_{u} a_{u}+b_{u}\right) I\left\{s \leqslant \sigma_{0} \leqslant u \leqslant t\right\} d u \mid \mathscr{F}_{s \wedge \sigma_{0}}\right) \\
& =\mathbf{E}\left(\int_{s}^{t}\left(\mathbf{E}\left(M_{u} \mid \mathscr{F}_{\sigma_{0}}\right) I\left\{\sigma_{0} \leqslant u\right\} a_{u}+b_{u}\right)\right. \\
& \left.\quad \times I\left\{s \leqslant \sigma_{0} \leqslant u \leqslant t\right\} d u \mid \mathscr{F}_{s \wedge \sigma_{0}}\right) \\
& =\mathbf{E}\left(\int_{s}^{t}\left(M_{\sigma_{0}} a_{u}+b_{u}\right) I\left\{s \leqslant \sigma_{0} \leqslant u \leqslant t\right\} d u \mid \mathscr{F}_{s \wedge \sigma_{0}}\right) \leqslant 0 .
\end{aligned}
$$

Здесь мы использовали тот факт, что $\left(M_{\sigma_{0}} a_{u}+b_{u}\right) I\left\{\sigma_{0} \leqslant u\right\}=$ $a_{u}\left(-b_{\sigma_{0}} / a_{\sigma_{0}}+b_{u} / a_{u}\right) I\left\{\sigma_{0} \leqslant u\right\} \leqslant 0$, а также равенство $\mathbf{E}\left(M_{u} \mid \mathscr{F}_{\sigma_{0}}\right) I\left\{\sigma_{0} \leqslant\right.$ $u\}=M_{\sigma_{0}} I\left\{\sigma_{0} \leqslant u\right\}$. Аналогично,

$$
\begin{aligned}
& \mathbf{E}\left(\int_{s}^{t}\left(M_{u} a_{u}+b_{u}\right) d u I\left\{\sigma_{0}<s\right\} \mid \mathscr{F}_{s \wedge \sigma_{0}}\right) \\
& \quad=\mathbf{E}\left(\int_{s}^{t}\left(\mathbf{E}\left(M_{u} \mid \mathscr{F}_{\sigma_{0}}\right) I\left\{\sigma_{0}<u\right\} a_{u}+b_{u}\right) d u I\left\{\sigma_{0}<s\right\} \mid \mathscr{F}_{s \wedge \sigma_{0}}\right) \\
& \quad=\mathbf{E}\left(\int_{s}^{t}\left(M_{\sigma_{0}} a_{u}+b_{u}\right) d u I\left\{\sigma_{0}<s\right\} \mid \mathscr{F}_{s \wedge \sigma_{0}}\right) \leqslant 0 .
\end{aligned}
$$

Значит, $\left\{\zeta_{s}, \mathscr{F}_{s \wedge \sigma_{0}}, 0 \leqslant s \leqslant T\right\}-$ супермартингал, причем $\zeta_{0}=0$, откуда для любого ограниченного момента остановки $\tau \mathbf{E} \zeta_{\tau} \leqslant 0$. В частности, если $\sigma_{0} \leqslant \tau \leqslant T$, то $\mathbf{E} \zeta_{\tau}=\mathbf{E} \int_{\sigma_{0}}^{\tau}\left(M_{u} a_{u}+b_{u}\right) d u \leqslant 0$. Теорема доказана.

Заметим, что капитал инвестора в момент $\sigma_{0}$ равен

$$
X_{\sigma_{0}}=\gamma e^{-\beta \sigma_{0}}\left[\frac{\left(a_{2}\left(\sigma_{0}\right)+\beta\right)\left(1-c_{\sigma_{0}}\right)}{\left(1-c_{\sigma_{0}}\right)\left(a_{2}\left(\sigma_{0}\right)-a_{1}\left(\sigma_{0}\right)\right)+c_{\sigma_{0}}^{\prime}}-1\right]>0,
$$

и, значит, «переключаясь» в момент $\sigma_{0}$, инвестору не нужно брать в долг.

(б) Пусть теперь инвестор платит цену в момент переключения $\tau$, если $\tau<T$ и $X_{\tau}>0$ или если $\tau=T$. Если же $\tau<T$ и $X_{\tau} \leqslant 0$, то он не 
переключается и не платит цену до момента $T$. Тогда задачей является нахождение $\sup _{\tau \leqslant T} \mathbf{E} \widehat{Y}_{\tau}$, где

$$
\begin{aligned}
\widehat{Y}_{t}= & {\left[\widetilde{X}_{t}\left(1-c_{t}\right)-\gamma e^{-\beta t}\right] \exp \left\{\int_{t}^{T} a_{2}(s) d s\right\} } \\
& \times\left(I\left\{t<T, \tilde{X}_{t}>\frac{\gamma e^{-\beta t}}{1-c_{t}}\right\}+I\{t=T\}\right) \\
& +\left[\tilde{X}_{T}\left(1-c_{T}\right)-\gamma e^{-\beta T}\right] I\left\{t<T, \tilde{X}_{t} \leqslant \frac{\gamma e^{-\beta t}}{1-c_{t}}\right\} .
\end{aligned}
$$

Обозначим $Z_{t}=\tilde{X}_{t}\left(1-c_{t}\right)-\gamma e^{-\beta t}$. Тогда

$$
\begin{aligned}
\mathbf{E} \widehat{Y}_{\tau}= & \mathbf{E} Z_{\tau} \exp \left\{\int_{\tau}^{T} a_{2}(s) d s\right\} I\left\{\tau<T, Z_{\tau}>0\right\} \\
& +\mathbf{E} Z_{T}\left(I\{\tau=T\}+I\left\{\tau<T, Z_{\tau} \leqslant 0\right\}\right) .
\end{aligned}
$$

Пусть $\sigma(\tau)=\tau I\left\{\tau<T, Z_{\tau}>0\right\}+T\left(I\{\tau=T\}+I\left\{\tau<T, Z_{\tau} \leqslant 0\right\}\right)$, тогда $\sigma(\tau)$ является моментом остановки, причем $\mathbf{E} \widehat{Y}_{\tau}=\mathbf{E} Y_{\sigma(\tau)}$. Следовательно, $\sup _{\tau \leqslant T} \mathbf{E} \widehat{Y}_{\tau}=\sup _{\sigma(\tau) \leqslant T} \mathbf{E} Y_{\sigma(\tau)} \leqslant \sup _{\tau \leqslant T} \mathbf{E} Y_{\tau}$, а значит, в этом случае инвестор не получает дополнительной выгоды.

3 а м е ч а и е 2. В данной статье рассматривается «последовательная» обработка двух активов с помощью процедуры переключения. «Параллельная» обработка двух активов, а именно, американский опцион купли с функцией выплаты, определяющейся максимумом из двух активов, рассмотрена в [7]. Отыскание оптимального момента остановки (без переключения) в аналогичной ситуации подробно рассмотрено B $[8]$.

\section{СПИСОК ЛИТЕРАТУРЫ}

1. Morimoto $H$. Optimal stopping and a martingale approach to the penalty method. Tohoku Math. J., 1982, v. 34, p. 407-416.

2. Факеев $A . \Gamma$. Об оптимальной остановке случайных процессов с непрерывным временем. - Теория вероятн. и ее примен., 1970, т. XV, № 2, с. 336-344.

3. Лunчер P. III., IIIиряев A. H. Статистика случайных процессов. М.: Наука, 1974, $696 \mathrm{c}$.

4. Деллашери K. Емкости и случайные процессы. М.: Мир, 1975, 192 с.

5. Jacka S. D. Local times, optimal stopping and semimartingales. - Ann. Probab., 1993, v. 21, № 1, p. 329-339.

6. Новиков A. A. О моментных неравенствах для стохастических интегралов. Теория вероятн. и ее примен., 1971, т. XVI, № 3, с. 548-551.

7. Brodie M., Detemple J. The valuation of American options on multiple assets. Math. Finance, 1997, v. 7, № 3, p. 241-286.

8. Duffie $D$. Dynamic Asset Pricing Theory. Oxford: Princeton Univ. Press, 1992, 300 p. 